\title{
Normal and tangential oil film stiffness of modified spur gear with non-Newtonian elastohydrodynamic lubrication
}

\author{
Changjiang Zhou ${ }^{\mathrm{a},}$, Zeliang Xiao ${ }^{\mathrm{a}}$, Siyu Chen ${ }^{\mathrm{b}}, \mathrm{Xu}_{\mathrm{Han}}{ }^{\mathrm{a}}$ \\ ${ }^{a}$ State Key Laboratory of Advanced Design and Manufacturing for Vehicle Body, Hunan University, Changsha, 410082, PR China \\ ${ }^{\mathrm{b}}$ State Key Laboratory of High Performance Complex Manufacturing, Central South University, Changsha, 410083, PR China
}

\begin{abstract}
:
A new normal stiffness model of the oil film is established by the viscous-elastic fluid between a spur gear drive being equivalent to a massless spring element, and the tangential stiffness model is proposed according to the hypothesis of equal shear stress on laminar element surfaces. Effects of operating parameters (contact force and rotation speed) and geometry parameters (module, tooth number, pressure angle and modification) on the oil film stiffness from the root to the tip are investigated. The results show that the amplitude and fluctuation of the stiffness are closely related to the shear rate, effective viscosity and curvature radii. It is indicated that the rational parameter match is valid in mesh impact reduction and stationarity enhancement.
\end{abstract}

Keywords: normal and tangential stiffness; oil film; non-Newtonian EHL; modified spur gear.

\section{Introduction}

Lubrication becomes an increasingly significant issue for a gear drive under harsh working condition or in the demand for higher performance [1], and the elastohydrodynamic oil film between the mesh teeth is a crucial factor in friction and wear reduction [2]. In addition, the gear contact stiffness along the normal direction is concerned in dynamics, which has an important effect on the mesh impact, vibration and even the contact fatigue lives for a gear transmission system [3].

Gear lubrication has been studied after the numerical elastohydrodynamic lubrication model developed [4]. The non-Newtonian behaviors were investigated based on an EHL model of spur gear by Larsson [5], and the transient thermal effect was investigated at a generic elastohydrodynamic line contact [6]. As for non-Newtonian lubricant, it was notable that Ree-Eyring model failed to describe large shear rate, which was pointed out by Khonsari and Hua [7]. Later, Khonsari et al. analyzed the features of non-Newtonian fluid models in different boundary conditions and also discussed the validity of various rheology models over a wide range of shear rate [8]. Furthermore, Khonsari studied the effect of starvation on traction and film thickness behavior in thermo-EHL line contacts with Carreau viscosity model [9]. It was shown in published papers that the Ree-Eyring model was suitable for non-Newtonian lubricant with medium shear rate. Venner investigated the roughness amplitude reduction for EHL point contacts by Ree-Eyring model [10] and Liu studied starved lubrication of a spur gear in Ree-Eyring fluid lubrication [11]. Recently, Kahraman and Lubrecht investigated oil film damping based on Ree-Eyring model $[12,13]$.

Recently, a number of new processes have emerged in EHL theory and its applications. Liu et al studied the effects of starved lubrication on film thickness, coefficient of friction, and temperature rise in spur gear drive [11]. Venner et al predicted oil film thickness for the isoviscous and piezoviscous cases at circular contact and explained the reason that the impact and the rolling contact yielded similar film thickness and pressure distributions [14]. Habchi et al investigated the effects of thermo-mechanical properties of coatings on friction in elastohydrodynamic contacts, and studied the underlying physical mechanisms governing friction variations [15]. As for the micro-EHL on rough surface, Thakre et al discussed the micro-EHL behavior of seven different ionic liquids in textured surface of concentrated engineering contacts and benchmarked the performance of ionic liquids against smooth surface and Newtonian rheology [16]. Masjedi et al investigated the effect of surface roughness in point-contact EHL and derived the formulas for predicting the central film thickness, minimum film thickness, and the asperity load ratio [17].

The oil film normal stiffness has attracted attention from many researches with the development of EHL theory [18]. In gear dynamics, the contact stiffness of mesh teeth with lubricant was determined by experience [19]. However, the mesh time-varying stiffness of oil film was a critical factor for revealing the dynamic response of gear drive [13]. Qin et al studied the normal stiffness of elastohydrodynamic line contact with various radii of curvature,

*Corresponding author

E-mail address: yangtsezhou@ @nu.edu.cn 


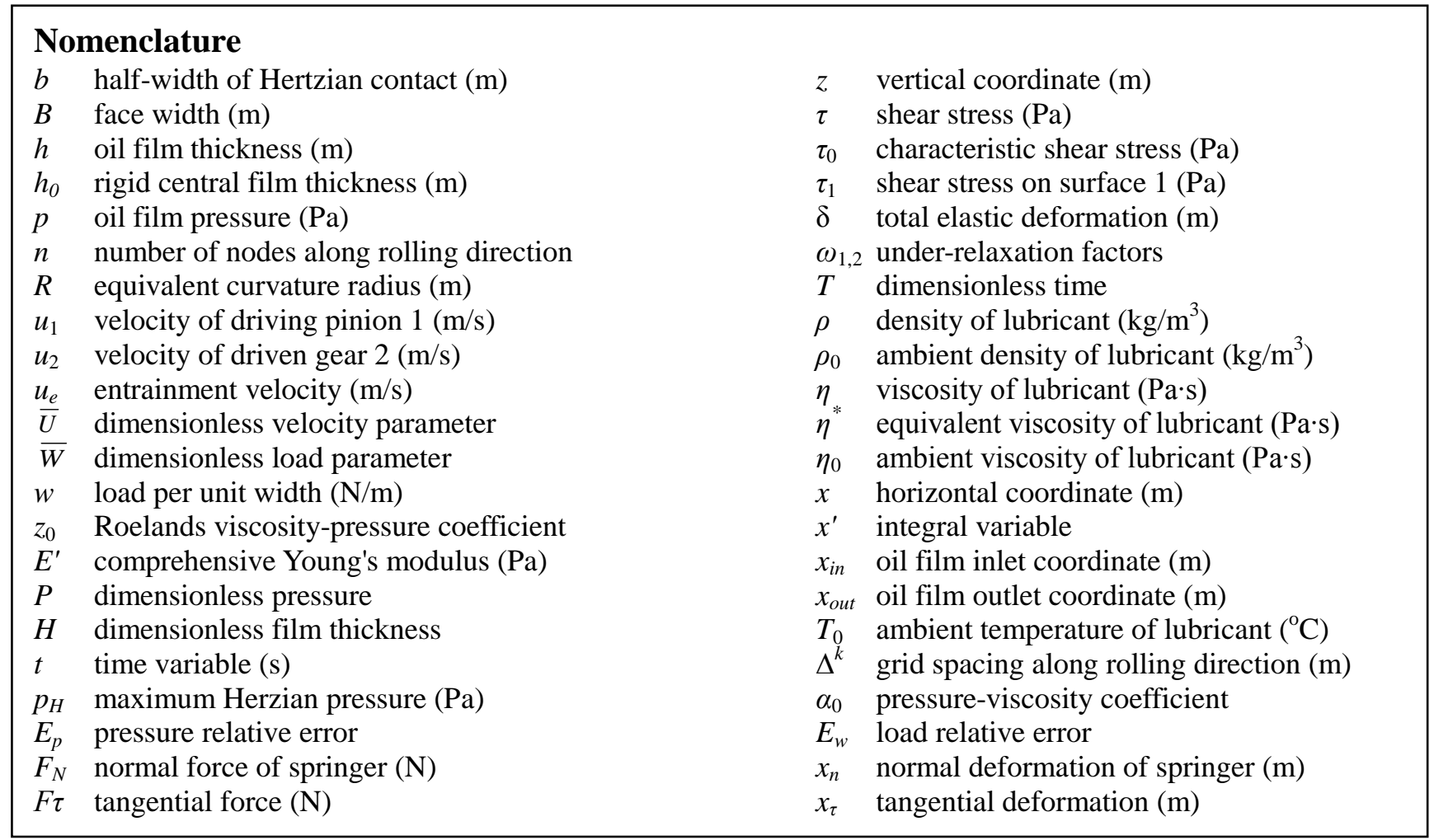

loads and entrainment velocities, and also investigated the critical condition in which the elastohydrodynamic stiffness could be approximated by Hertzian stiffness [20]. Daniel et al studied the lateral contact stiffness of asperities in the elastic deformation stage of asperities [21]. Then, Shi et al investigated the tangential contact stiffness of cylindrical according to the fractal theory [22]. Recently, the normal lubricant stiffness and structural damping based on an elastohydrodynamic lubrication line contact-vibration model were calculated and their impacts on gear dynamics were investigated [23].

The effects of EHL on gear drive have widely been focused, and the normal stiffness of the lubricant has also been concerned in gear dynamics. However, the mechanism of oil film stiffness underlying the mesh impact and transmission stationarity is still in its infancy. Theoretical EHL models have been developed to predict oil pressure and film thickness, but the researches on the normal and tangential stiffness are lacking. As a matter of fact, the total load is not along the normal direction if the friction force of the mesh teeth or shear film is included, and then the oblique impact is taken place on the contact profile. The aim of this paper is to study the normal and tangential lubricant stiffness models of unmodified and modified spur gears, and the current oil film stiffness models are established in fully flooded lubrication, while the contact surface stiffness and its effect on oil film stiffness were not included. Factually, the asperity contact has a significant influence on the oil film stiffness in mixed lubrication contact [24]. The variation of oil film stiffness with operating parameters (contact force and rotation speed) and geometry parameters (module, tooth number, pressure angle and modification). In order to clarify the function mechanism of the lubricant and gear parameters, effects of viscosity, rheological behavior and curvature radii of contact bodies on the oil film stiffness were to studied, which is helpful to explore a method for mesh impact reduction and stationarity enhancement by rationally matching the parameters.

\section{Kinematic equations}

\subsection{Generalized Reynolds equation}

The pressure distribution taking the non-Newtonian effect into account in the one-dimensional contact area is governed by the generalized Reynolds equation given by Yang and Wen [25].

$$
\frac{\partial}{\partial x}\left[\varepsilon \frac{\partial p}{\partial x}\right]=12 u_{e} \frac{\partial\left(\rho^{*} h\right)}{\partial x}
$$


where,

$$
\begin{aligned}
& \varepsilon=\left(\frac{\rho}{\eta}\right)_{e} \frac{h^{3}}{\lambda}, \lambda=\frac{3}{4} \pi^{2} \frac{\bar{U}}{\bar{W}^{2}} \\
& \left(\frac{\rho}{\eta}\right)_{e}=12\left(\eta_{e} \rho_{e}^{\prime} / \eta_{e}^{\prime}-\rho_{e}^{\prime \prime}\right) \\
& \rho^{*}=\left[\rho_{e}^{\prime} \eta_{e}\left(u_{2}-u_{1}\right)+\rho_{e} u_{1}\right] / u_{e} \\
& \rho_{e}=\frac{1}{h} \int_{o}^{h} \rho d z \quad \rho_{e}^{\prime}=\frac{1}{h^{2}} \int_{0}^{h} \rho \int_{0}^{z} \frac{d z^{\prime}}{\eta^{*}} d z \\
& \rho_{e}^{\prime \prime}=\frac{1}{h^{3}} \int_{0}^{h} \rho \int_{0}^{z} \frac{z^{\prime} d z^{\prime}}{\eta^{*}} d z \quad \frac{1}{\eta_{e}}=\frac{1}{h} \int_{0}^{h} \frac{d z}{\eta^{*}} \quad \frac{1}{\eta_{e}^{\prime}}=\frac{1}{h^{2}} \int_{0}^{h} \frac{z d z}{\eta^{*}}
\end{aligned}
$$

where $p, h$ and $\rho$ stand for the pressure, film thickness and density of the lubricant, respectively. $x$ and $z$ represent the coordinates along the moving direction and across the film. $u_{1}$ and $u_{2}$ are the velocities of the contact surfaces for the driving pinion and driven gear respectively, and $u_{e}$ is the entrainment velocity. As for boundary conditions, ambient pressure is prescribed at the inlet and outlet of the fluid domain. The corresponding abscissas, $x_{i n}$ and $x_{\text {out }}$ are located at $x_{i n}=-4.5 b$ and $x_{\text {out }}=1.5 b$, respectively. $b$ being the Hertzian contact half-width. In this paper, the shear rate of oil film is not large and Ree-Eyring model is applicable to a low-speed and mid-load spur gear drive. Therefore, the Ree-Eyring fluid model is utilized to describe the shear-thinning behavior of EHL lubricant and the non-Newtonian effect is taken into account by application of the generalized Reynolds equation in which the equivalent viscosity is employed. The equivalent viscosity of the Ree-Eyring fluid varied along and across the film is given as

$$
\eta^{*}=\eta\left(\frac{\tau}{\tau_{0}}\right) / \sinh \left(\frac{\tau}{\tau_{0}}\right),
$$

where $\tau$ and $\tau_{0}$ are the shear stress and characteristic shear stress of the fluid, respectively.

\subsection{Film thickness equation}

The film thickness equation in an EHL line contact problem is given by:

$$
h=h_{0}+\frac{x^{2}}{2 R}-\frac{2}{\pi E^{\prime}} \int_{x_{\text {in }}}^{x_{\text {out }}} p \ln \left(x-x^{\prime}\right) d x^{\prime},
$$

where $h_{0}$ is the spacing between the rigid contact bodies, and $R$ is the equivalent radius of curvature. The last term in Eq. (3) is the total elastic deflection of two contact bodies.

\subsection{Lubricant viscosity-pressure and density-pressure equations}

As for EHL, high contact pressure results in a large elastic deformation of the contact surfaces, and an enormous increase of the lubricant viscosity is caused by the deformation. Hence, the viscosity variation with contact pressure is of significant importance. In the present study, the viscosity-pressure model proposed by Roelands is used.

$\eta=\eta_{0} \exp \left\{\left(\ln \eta_{0}+9.67\right)\left[\left(1+5.1 \times 10^{-9} p\right)^{z_{0}}-1\right]\right\}$,

where $\eta_{0}$ is the viscosity of the lubricant under the ambient pressure. The density $\rho$ of the fluid is related to the local pressure in accordance with Dowson and Higginson's density-pressure rule, which is given as

$\rho=\rho_{0}\left[1+\left(0.6 \times 10^{-9} p\right) /\left(1+1.7 \times 10^{-9} p\right)\right]$, 
where $\rho_{0}$ is the density of the lubricant under the ambient pressure.

\subsection{Load balance equation}

The load per unit width acting in the contact domain has to be balanced by the elastohydrodynamic load. Thus, the load balance equation is given by

$\int_{x_{\text {in }}}^{x_{\text {out }}} p d x=w$,

where $w$ is per unit width load. The rigid separation is an unknown variable in each time step. It can be determined by solving the Reynolds equation with the load balance equation.

\subsection{Shear stress equation}

The lubricant shear stress of Ree-Eyring fluid is expressed as

$\tau=\tau_{1}+z \frac{\partial p}{\partial x}$,

$\tau_{1}=\tau_{0} \ln \frac{\sqrt{\left(u_{1}-u_{2}\right)^{2}+\left(F_{1}^{2}-F_{2}^{2}\right)}-\left(u_{1}-u_{2}\right)}{F_{1}+F_{2}}$,

The integral functions used in Eq. (8) are defined as follows

$$
\begin{aligned}
& F_{1}=\int_{0}^{h} \frac{\tau_{0}}{\eta} \cosh \left(\frac{z}{\tau_{0}} \cdot \frac{\partial p}{\partial x}\right) d z \\
& F_{2}=\int_{0}^{h} \frac{\tau_{0}}{\eta} \sinh \left(\frac{z}{\tau_{0}} \cdot \frac{\partial p}{\partial x}\right) d z
\end{aligned}
$$

where $\tau_{1}$ is the shear stress on the contact surface 1 .

\subsection{Velocity and velocity gradient equations}

The velocity gradient of a non-Newtonian fluid across the film can be determined by the following equation:

$$
\frac{\partial u}{\partial z}=\frac{\tau}{\eta^{*}},
$$

Integrating this equation with respect to $z$, the velocity is finally derived as

$$
u=u_{1}+\int_{0}^{z} \frac{\partial u}{\partial z^{\prime}} d z^{\prime},
$$

\section{Models and solution}

\subsection{Oil film normal stiffness}

In a mesh cycle, the contact force, curvature radius and entrainment speed vary along line of action (LOA) from the root to the tip. At a certain mesh position, the oil film pressure, $p(x, t)$, including the non-Newtonian effect, is 
obtained by Eq. (1), and the oil film thickness, $h(x, t)$, is calculated by Eq. (2). The compression load of per unit width, $w(t)$, at an elastohydrodynamic line contact is shown in Fig. 1. If a contact force increment, $\Delta w(t)$, is given, the oil pressure increment, $\Delta p(x, t)$, and the compression increment of the oil film thickness, $\Delta h(x, t)$, are attained. As a viscous-elastic fluid, the oil film between the mesh teeth is equivalent to a massless spring element in Fig. 2 . Thus the oil film normal stiffness of an elastohydrodynamic line contact for a spur gear drive is defined as [20],

$$
k_{n}(t)=\frac{\Delta F_{n}(t)}{\Delta x_{n}(t)}=\frac{B \times \Delta^{k}(t) \sum \Delta p(x, t)}{\frac{1}{n} \sum \Delta h(x, t)},
$$

where $B$ is the face width, $\Delta$ is a symbol representing increment and $\Delta^{\mathrm{k}}$ is the grid spacing in the rolling direction.

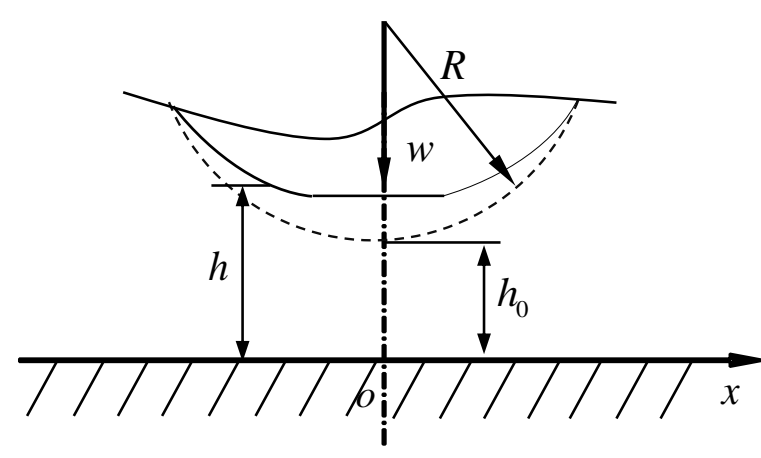

Fig.1 Line contact model

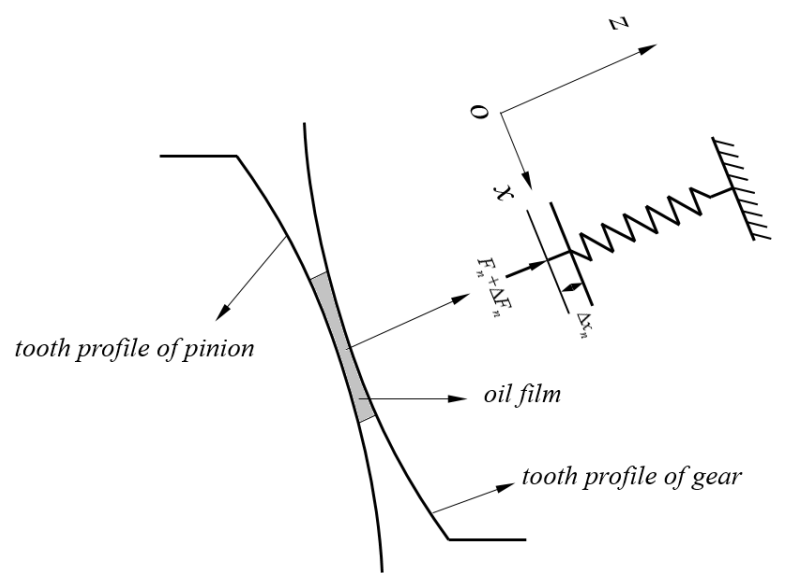

Fig.2 Equivalent spring model of the normal stiffness

As for a spur gear, the oil film normal stiffness is a time-varying period variable along LOA. The reason is that the oil pressure and film thickness vary with the mesh position, where the contact force, radius of curvature and entrainment speed are changed.

\subsection{Oil film tangential stiffness}

As the lubricant flows through the laminar contact domain, the tangential displacement of oil film is caused by the shear stress on adjacent layers of the viscous lubricant. The oil film tangential stiffness $k_{\tau}(t)$ can be calculated according to the first derivative of the tangential force with respect to tangential displacement [22]. The shear stress of Ree-Eyring fluid, $\tau(x, z, t)$, is obtained by Eq. (7) and the shear rate is given by Ref.[26].

$\dot{\gamma}=\frac{\tau_{0}}{\eta} \sinh \left(\frac{\tau}{\tau_{0}}\right)=\frac{\partial u}{\partial z}$,

Integrating the equation with respect to $t$, the shear strain is finally derived as

$\gamma=\int_{0}^{t_{1}} \frac{\partial u}{\partial z} d t$

where $t_{1}$ denotes the time of the fluid across the contact domain.

So that

$\Delta x_{\tau}=\gamma \Delta z$

The shear stress gradient with respect to $\mathrm{z}$ can be neglected because it is much smaller than the shear stress. The equivalent tangential stiffness model of oil film is shown in Fig. 3. The element drawn in dotted line is loaded on shear force, $F_{\tau}(t)$, and the element drawn in solid line is loaded on the shear force increment, $\Delta F_{\tau}(t)$, and $\Delta x_{\tau}(t)$ is the 
increased tangential displacement. Therefore, the oil film tangential stiffness of elastohydrodynamic line contact for a spur gear drive is defined as

$$
k_{\tau}(t)=\frac{\Delta F_{\tau}(t)}{\Delta x_{\tau}(t)}=\frac{B \times \Delta^{k}(t) \sum \Delta \tau(x, z, t)}{\frac{1}{n} \sum\left(\left[\int_{0}^{t_{1}} \dot{\gamma}(x, z, t) d t\right] \Delta z\right)},
$$

where $\Delta z$ is the dimension of tangential stiffness model at the direction across the oil film.

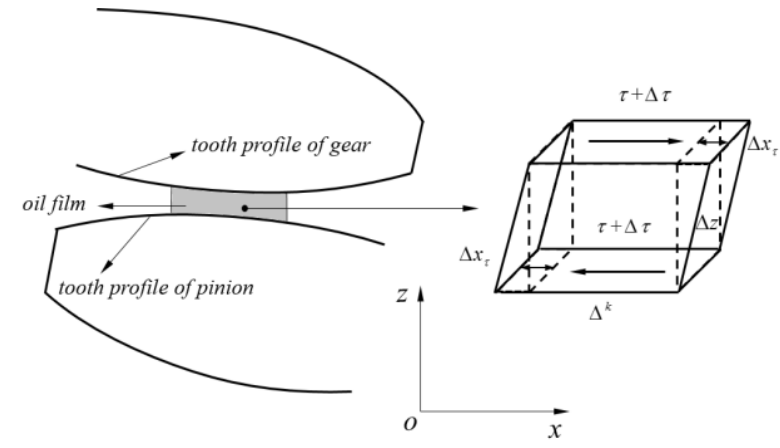

Fig.3 The equivalent tangential stiffness model

\subsection{Numerical solution}

The EHL equations and boundary conditions are written into dimensionless form to facilitate the numerical calculation, so that the following dimensionless variables are utilized.

$$
\begin{aligned}
& X=x / b, X_{\text {in }}=x_{\text {in }} / b, X=x_{\text {out }} / b, H=h R / b^{2}, Z=z / h, P=p / p_{H}, T=t u_{e} / b, \bar{\rho}=\rho / \rho_{0}, \\
& \bar{\eta}^{*}=\eta^{*} / \eta_{0}, \bar{\eta}=\eta / \eta_{0}, \bar{\tau}=\tau / p_{H}, \overline{\tau_{1}}=\tau_{1} / p_{H}, \overline{\tau_{0}}=\tau_{0} / p_{H}, \bar{u}=u / u_{e}, \overline{u_{1}}=u_{1} / u_{e}, \overline{u_{2}}=u_{2} / u_{e}, \\
& \bar{W}=w /\left(E^{\prime} R\right), \bar{U}=u_{e} \eta_{0} /\left(E^{\prime} R\right)
\end{aligned}
$$

In order to calculate the oil film normal and tangential stiffness and to investigate the effects of operating and geometry parameters on the stiffness, the numerical scheme is to update the pressure and film thickness independently and to iterate between Reynolds equation and the film thickness equation until the full numerical solution of relevant variables is obtained. The pressure distribution is obtained by the multi-grid method [27]. In pressure iterative process, 6 grid levels with 961 equidistant nodes on the finest grid level at the rolling direction $(x$ direction) are utilized and the oil film gap ( $z$ direction) is divided into 51 equidistant nodes. A mesh cycle is divided into a uniform mesh consisting of 100 nodes. The calculation domain for the pressure is $x_{i n}=-4.5 b, x_{\text {oul }}=1.5 b$. Parameters of the spur gear drive and the lubricant are listed in Table 1. At the lowest point of single tooth contact (LPSTC) and the highest point of single tooth contact (HPSTC), sharp changes for the load carried per unit width are in existence. The contact force distribution along LOA is provided by the method of Ref. [28].

\section{Table 1}

Gear geometry and lubricant parameters.

\begin{tabular}{llll}
\hline Item & Parameter & Item & Parameter \\
\hline Number of teeth & $N_{1}=51, N_{2}=152$ & Ambient viscosity of lubricant & $\eta_{0}=0.08 \mathrm{~Pa} \cdot \mathrm{s}$ \\
Module & $m=0.0025 \mathrm{~m}$ & Pressure-viscosity coefficient & $a_{0}=2.19 \times 10^{-8} \mathrm{~Pa}^{-1}$ \\
Pressure angle & $\alpha=20^{\circ}$ & Comprehensive Young's modulus & $E^{\prime}=2.28 \times 10^{11} \mathrm{~Pa}$ \\
Face width & $B=0.03 \mathrm{~m}$ & Characteristic shear stress & $\tau_{0}=10 \mathrm{MPa}$ \\
Input rotation speed & $n_{1}=500 \mathrm{r} / \mathrm{min}$ & Dimensionless material parameter & $G=5000$ \\
Load increment per unit width & $w=140 \mathrm{kN} / \mathrm{m}$ & Ambient oil temperature & $T_{0}=30^{\circ} \mathrm{C}$ \\
\hline
\end{tabular}


For the pressure convergence, the following criterion is employed:

$E_{p}=\frac{\sum\left|P_{i}-\bar{P}_{i}\right|}{\sum\left|P_{i}\right|} \leq 0.001$

If the convergence is not achieved, the pressure is updated as

$\tilde{P}_{i}=P_{i}+\omega_{1} \delta_{i}$

where $\tilde{P}_{i} \quad P_{i}, \bar{P}_{i}$ are the new updated pressure, the obtained pressure in the current iteration process and the pressure in the last iteration process, respectively. In the pressure updated process, the Gauss-Seidel iterative method is applied and $\omega_{1}$ is the under-relaxation factor with the usual range 0.2-1.2 which usually should be chosen to a minor value as the load is very large. $\delta_{i}$ represents the correction coefficient of oil film pressure.

For the load convergence, the following criterion is utilized:

$$
E_{w}=\frac{\left|0.5 \Delta^{k} \sum_{j=1}^{n-1}\left(P_{j}+P_{j+1}\right)-\pi / 2\right|}{\pi / 2}<0.001
$$

If the load does not converge, the gap is adjusted according to the evaluated unbalanced load, according to the following relationship:

$$
\tilde{H}_{0}=H_{0}+\omega_{2}\left(0.5 \Delta^{k} \sum_{j=1}^{n-1}\left(P_{j}+P_{j+1}\right)-\pi / 2\right)
$$

where $\tilde{H}_{0}$ is the new updated oil film gap, and $\omega_{2}$ is the under-relaxation factor with the usual range $0.02-0.4$. Generally, the factor is chosen as a minor value if the load is very large.

As for unmodified gear and pinion in Table 1, the sharp change of the load on the tooth profile is observed in single-to-double or double-to-single tooth contact region in Fig. 4. To reduce transmission errors and mesh impact, most of industrial gears have to be modified through removal of the additional material and tooth deflection is compensated. A tip relief and profile crown modification such as circular profile modification are adopted widely [23]. The modification methods are also applied to the driving pinion and driven gear in present study. At the same input load of $150 \mathrm{kN} / \mathrm{m}$, the variations of the load per unit width along LOA for standard and modified spur gears are shown in Fig. 4.

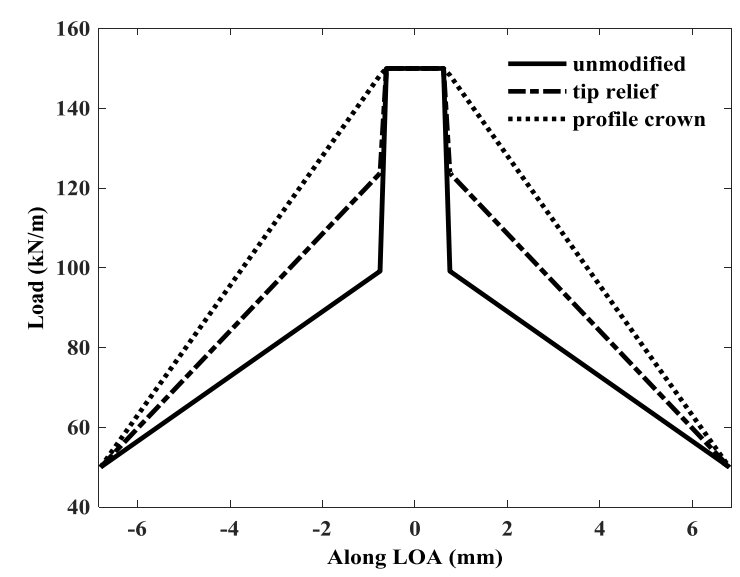

Fig.4 Load distribution of standard and modified spur gears

The flow chart representing the solution of oil film normal and tangential stiffness is given in Fig. 5. 


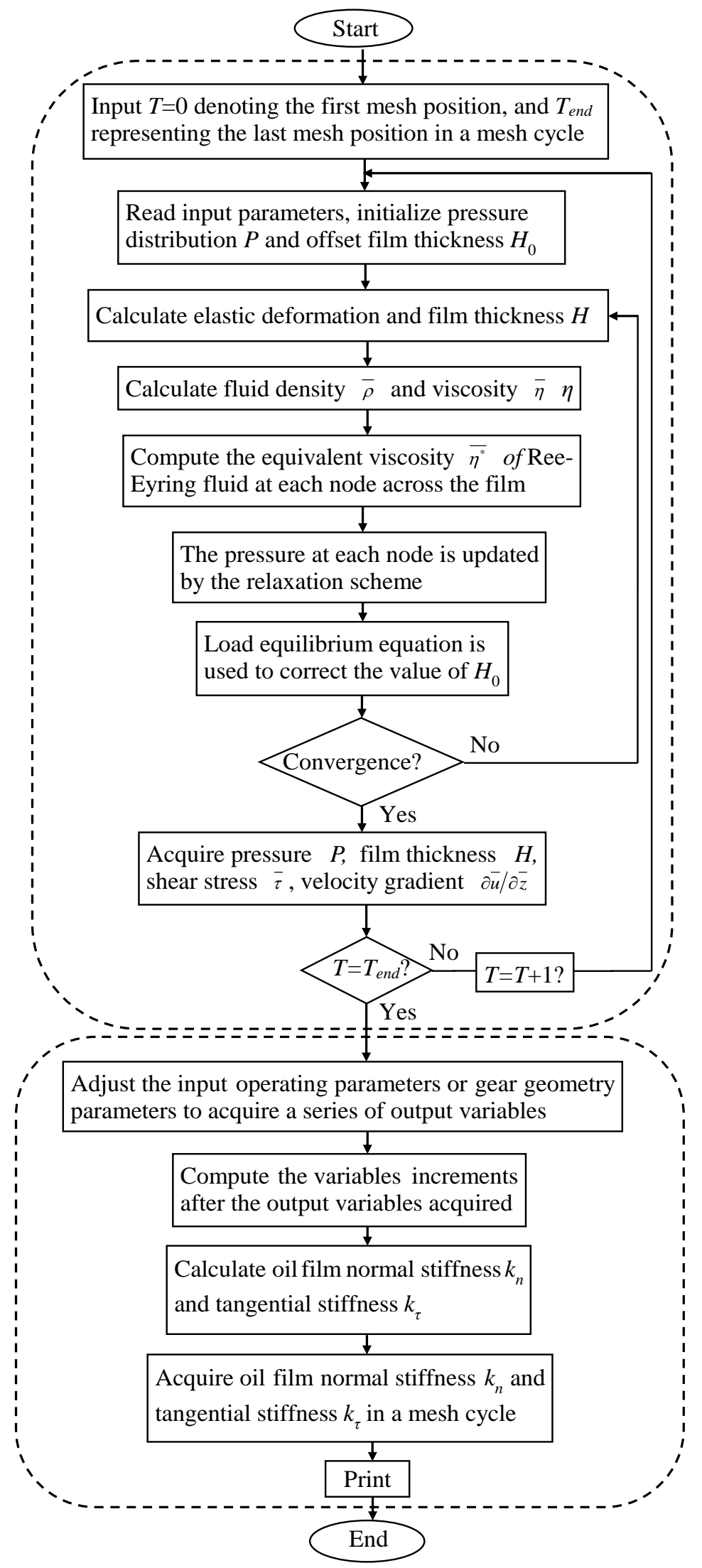

Fig. 5. Numerical computation procedure of calculating the oil film stiffness 
According to the flow chart in Fig.5, the curves of oil film pressure, thickness and traction at three characteristic locations (engaging-in point, pitch point and recession point) are plotted to verify the validity of the numerical procedure. As shown in Fig.6, the pressure falls down as the oil film thickness becomes thicker, and the pressure distribution in engaging-in or recession point is less than that in pitch point, where the contact force is reached the peak. In the most contact domain, the oil film pressure is approximate to Hertzian contact pressure without lubrication. The second pike of oil pressure is clearly observed on the pressure curve of the pitch point, which is an important feature in EHL contact. It can be explained that the flow of viscous-elastic lubricant is hindered by sharp divergent gap shape, which results in the second pressure spike described in previous researches [12,29]. The oil film pressure and thickness in engaging-in point are similar to those in recession point due to the same contact force. In addition, the traction distributions are similar to the pressure distributions in the same positions, but the traction magnitude order is far less than that of oil pressure, which results in the friction coefficient being far less than 1.0. Negative value means the direction of shear stress is inverse of slide velocity.
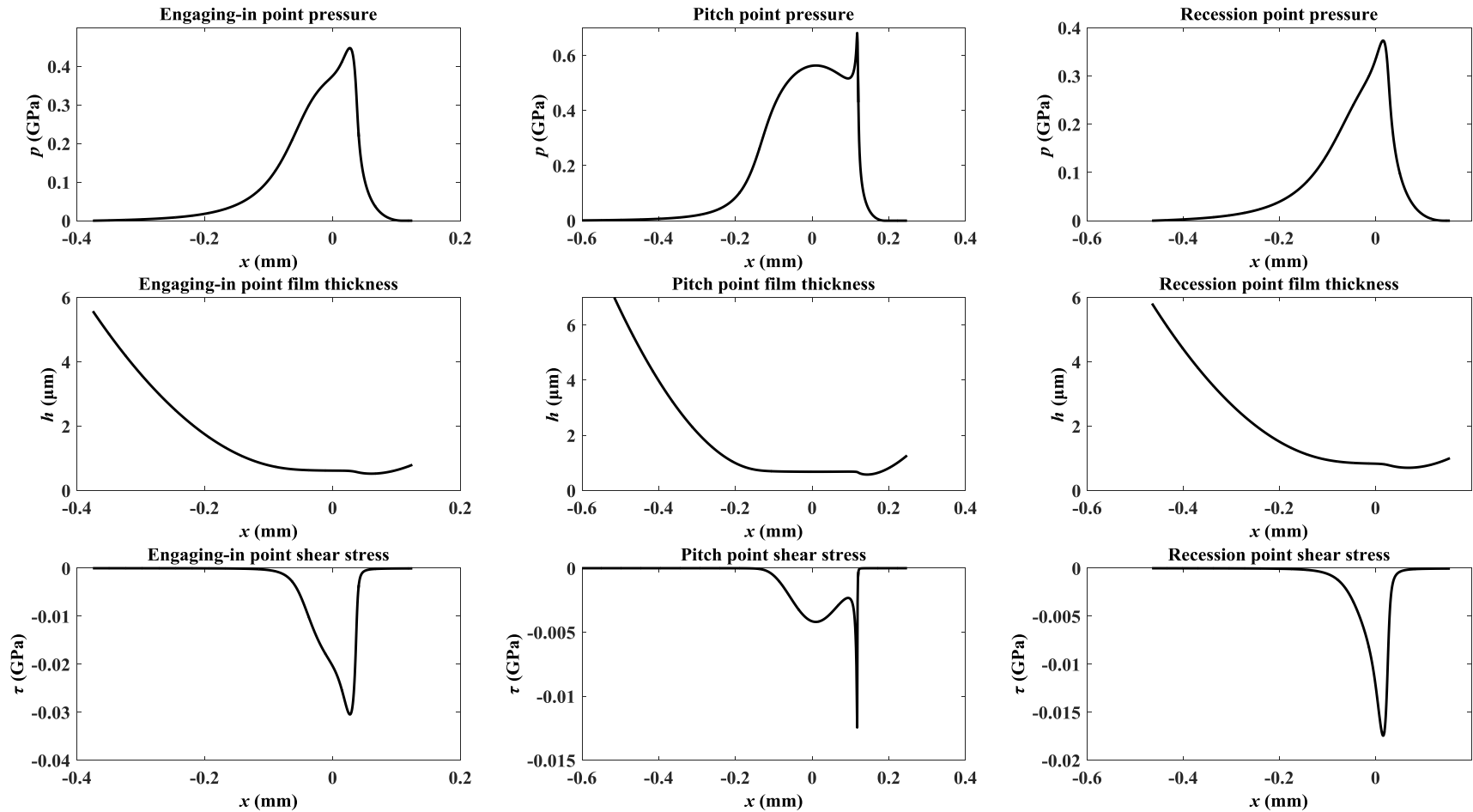

Fig. 6. Oil film pressure, film thickness and traction distributions at three characteristic locations

\section{Results and discussion}

\subsection{Effect of contact force}

The oil film normal and tangential stiffness become larger as increase of the load along LOA in Fig.7, since the compression increment of film thickness is smaller than the oil pressure increment. In Fig.7 (a), the normal stiffness is slightly larger at LPSTC than HPSTC, and it mildly decreases from the peak at the single-tooth contact, under the effect of entrainment velocity and curvature radius. In Fig.7 (b), the tangential stiffness is symmetrical about the pitch point and slightly increases in the double-teeth contact region as the contact force is raised, whereas it sharply increases as the contact moves closer towards the pitch point near the middle of single-tooth contact domain, where the maximum tangential stiffness is reached. The sharp increase of the tangential stiffness is caused by two primary reasons [12]:1) the effective viscosity of the lubricant is enhanced as the film pressure is larger near the pitch point; 2) the non-Newtonian behavior of the lubricant is reduced as the slide-roll ratio is close to zero at the pitch point and the 
velocity gradient across oil film reached the minimum value. To sum up, the oil film normal and tangential stiffness become larger as the contact force increases due to the effect of pressure on lubricant equivalent viscosity. However, the fluctuation of the normal stiffness becomes smaller in the single-to-double and double-to-single tooth contact regions as rise of the load, whereas the tangential stiffness increment is much large as the oil pressure is raised in the single-tooth contact region. Factually, the smaller fluctuation of normal stiffness leads to the improvement of impact resistance and shock absorption of oil film, and the larger tangential stiffness results in the loss of fluidity.

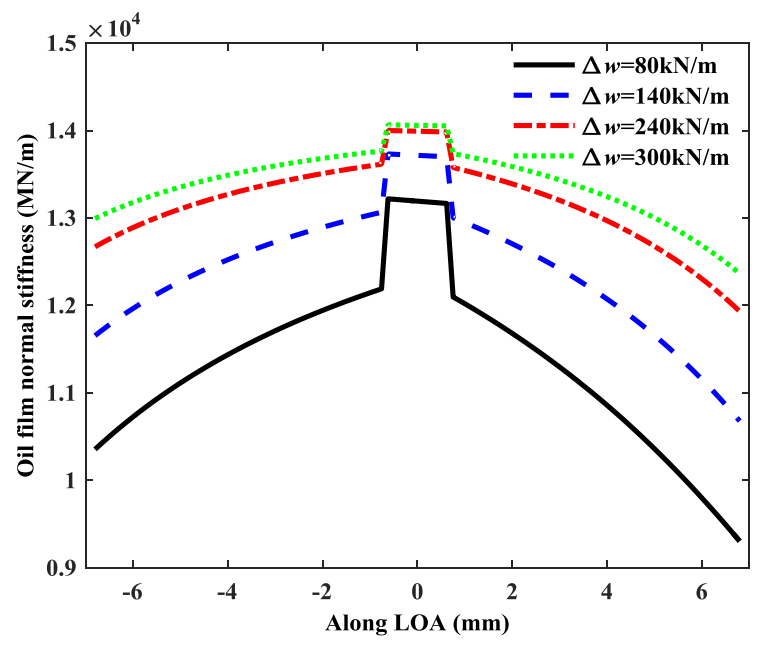

(a)

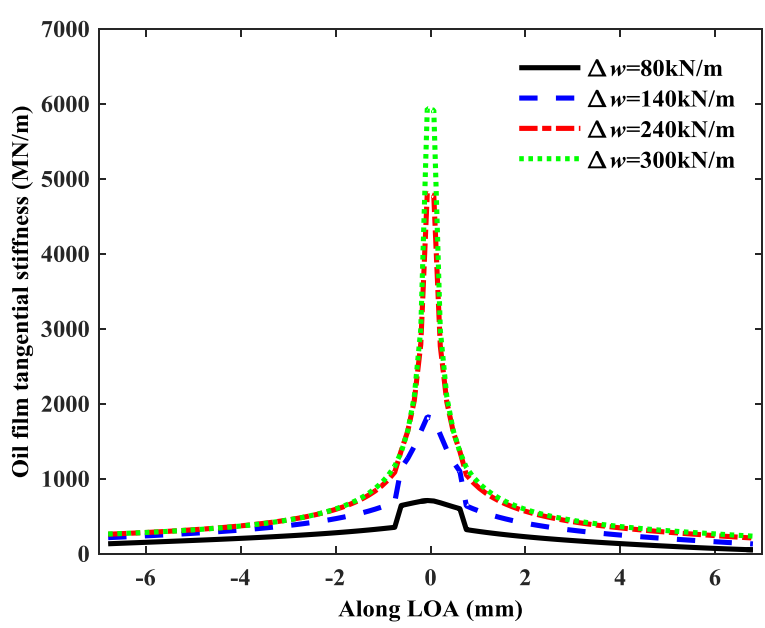

(b)

Fig. 7. Variation of the oil film normal and tangential stiffness with contact force

\subsection{Effect of rotation speed}

As shown in Fig. 8, the oil film normal stiffness decreases as the rotation speed is raised in a mesh cycle, whereas the tangential stiffness becomes larger. The decrease of the normal stiffness is related to the increase of the entrainment velocity, which becomes greater as rise of the rotation speed. Moreover, the shift of the normal stiffness varied with rotation speed is consistent with the result from Ref. [20]. It is shown in Fig. 8 (b) that the entrainment velocity plays an important role on the shear rate at tangential direction. As the same contact width covered, the integral time becomes shorter when the rotation speed is up, which leads to the decrease of the shear rate. With the decrease of oil film normal stiffness, the impact resistance of gear pair and the shock absorption of oil film would be enhanced. However, the fluidity is decreased as rise of the tangential stiffness, especially in single-tooth mesh region.

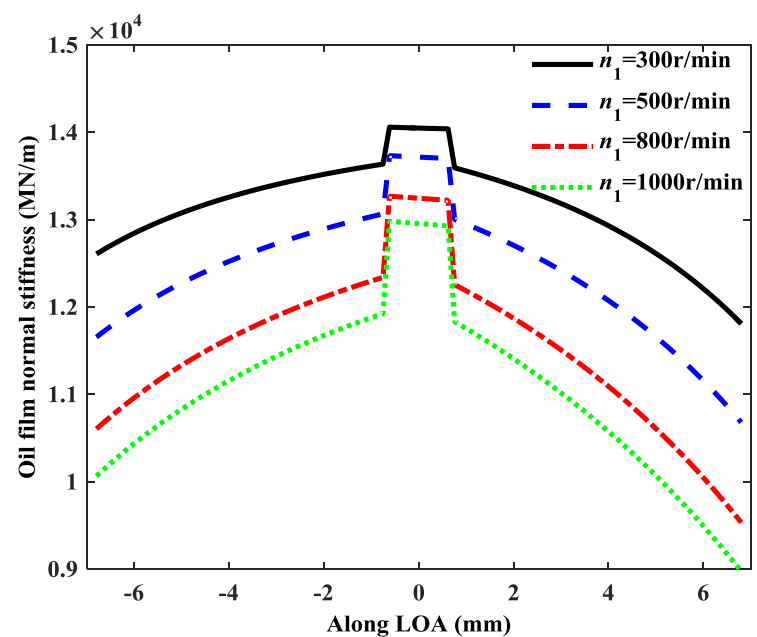

(a)

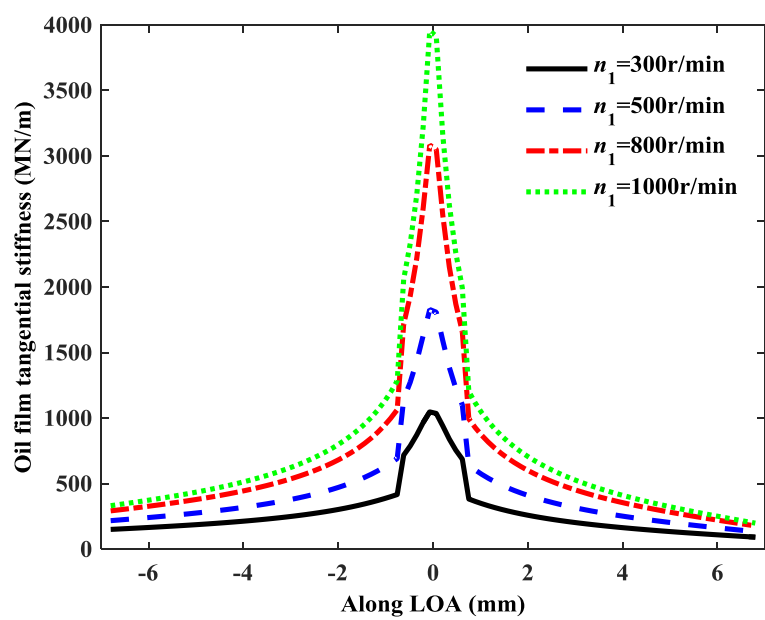

(b)

Fig. 8. Variation of the oil film normal and tangential stiffness with rotation speed 


\subsection{Effect of module}

In a mesh cycle, the oil film normal and tangential stiffness become smaller as the module is increased (Fig. 9). However, the tangential stiffness sharply decreases in single-tooth mesh region as increase of the module, which indicates that the radius of curvature plays an important role on the shear rate at tangential direction. The sharp decrease of the tangential stiffness can be explained as below:1) the radius of curvature becomes larger as the increase of module, which makes the contact region wider at the same contact force and rotation speed; 2) the longer integral time is needed when the wider contact region is covered, which results in increase of the shear rate. As a result, the considerable fluctuation of the tangential stiffness is caused by a slight change of the module.

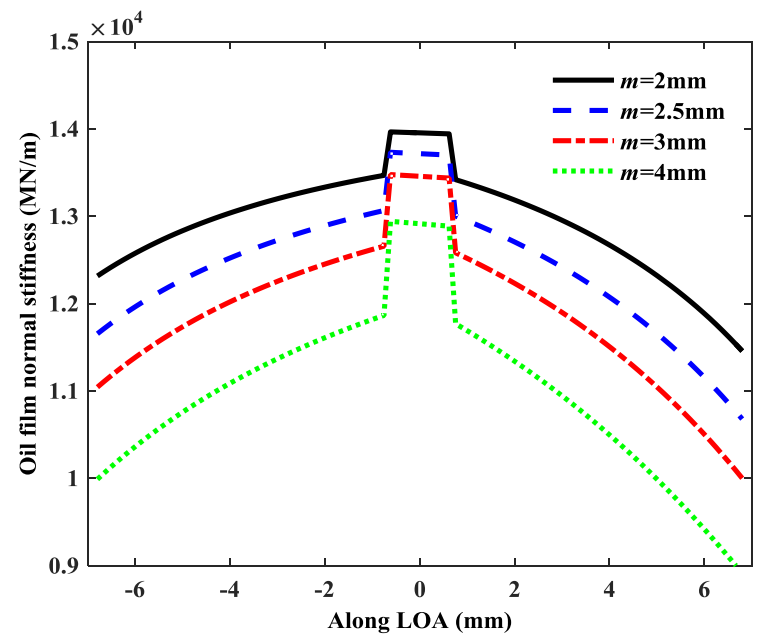

(a)

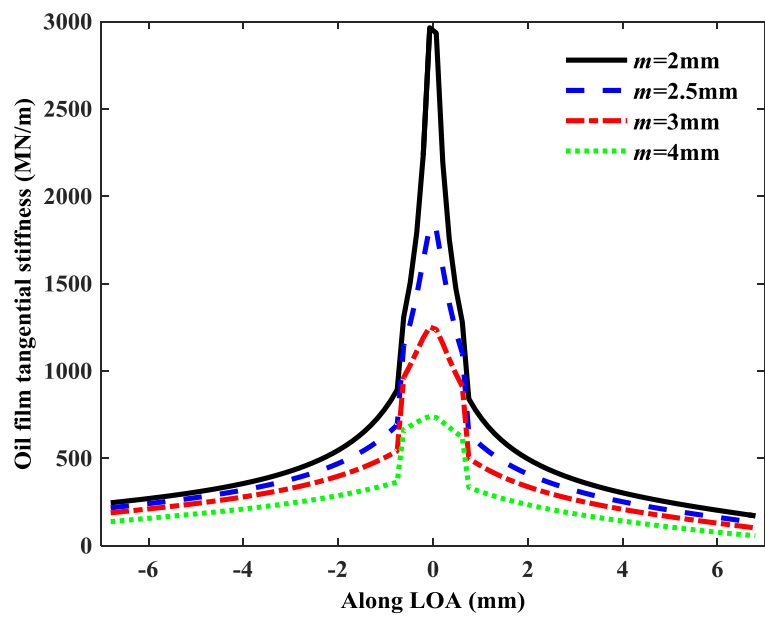

(b)

Fig. 9. Variation of the oil film normal and tangential stiffness with module

\subsection{Effect of tooth number}

As shown in Fig. 10, the oil film normal and tangential stiffness along LOA fall down as increase of tooth numbers. The curvature radius of tooth profile becomes larger as the tooth numbers are raised, and the smaller stiffness can be obtained by increasing the curvature radii of contact bodies. It is clearly shown in Fig. 10 (b) that the tangential stiffness rapidly decreases in the single-tooth mesh region and is almost unchanged in the double-teeth contact region when the tooth numbers become greater. Therefore, it indicates that the tangential stiffness increment of oil film is closely relative to the contact domains (single-tooth or double-teeth mesh region).

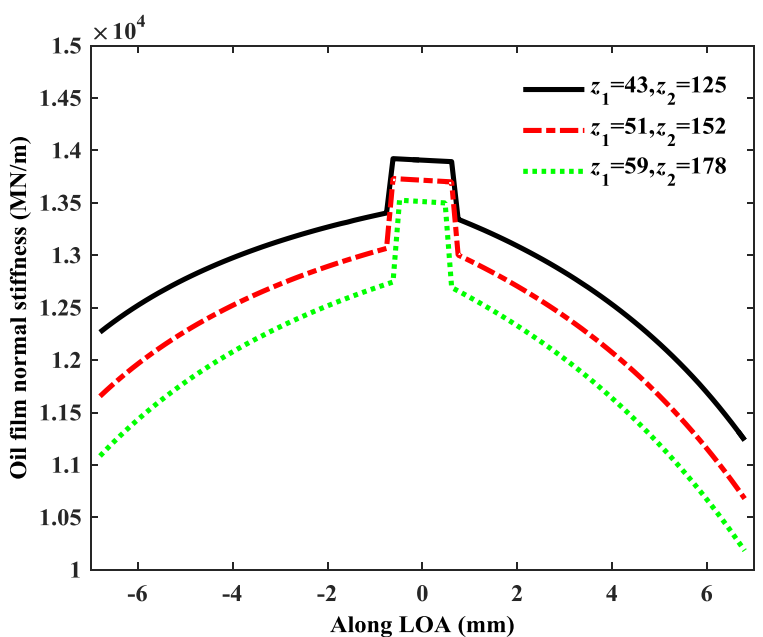

(a)

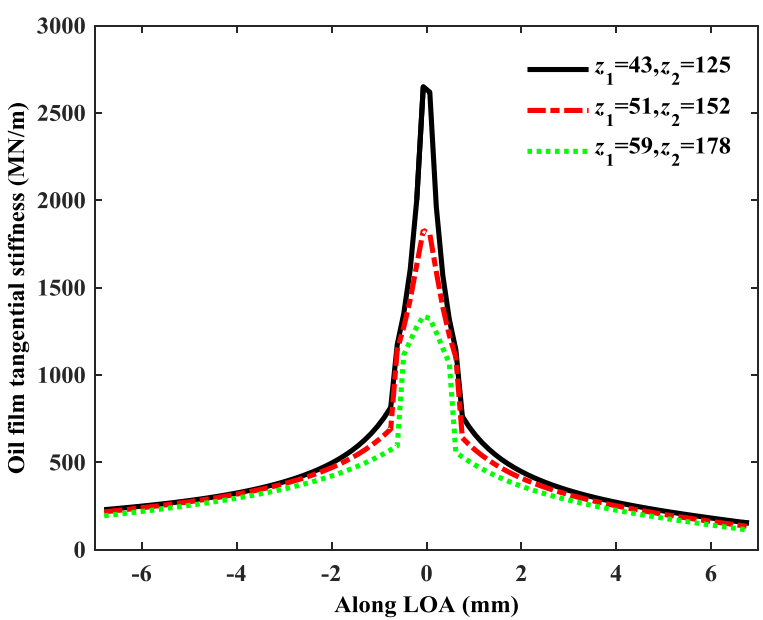

(b) 
Fig. 10. Variation of the oil film normal and tangential stiffness with tooth number

\subsection{Effect of pressure angle}

The oil film normal and tangential stiffness become smaller as increase of the pressure angle in Fig. 11. As the pressure angle is bigger, the tooth profile becomes sharper from HPSTC to the tip and it becomes thicker from LPSTC to the root. The bigger pressure angle results in the enhancement of tooth bending and contact strength. However, the single-tooth contact region is broadened and the contact ratio becomes smaller as the pressure angle is raised, and the transmission stationarity is weakened. But the peak of oil film stiffness is cut down owning to the bigger pressure angle, especially for the tangential stiffness, which is valid in mesh impact reduction.

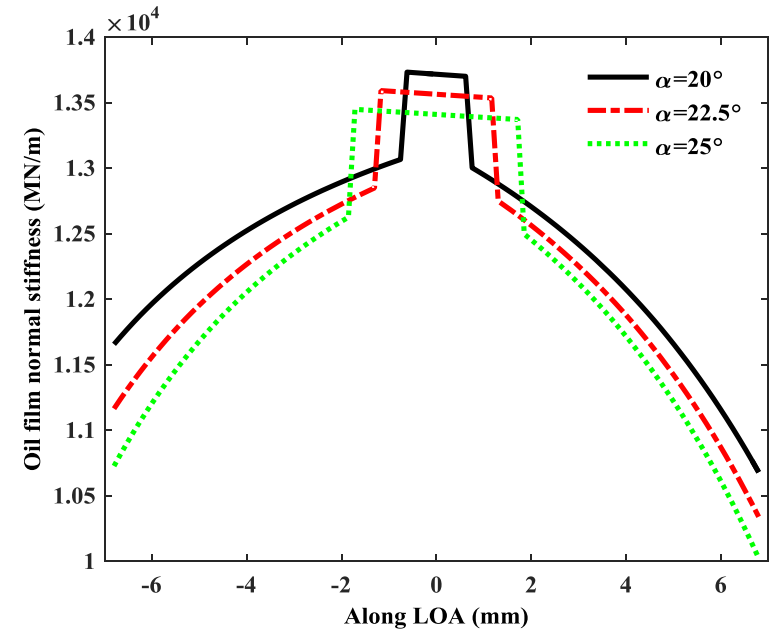

(a)

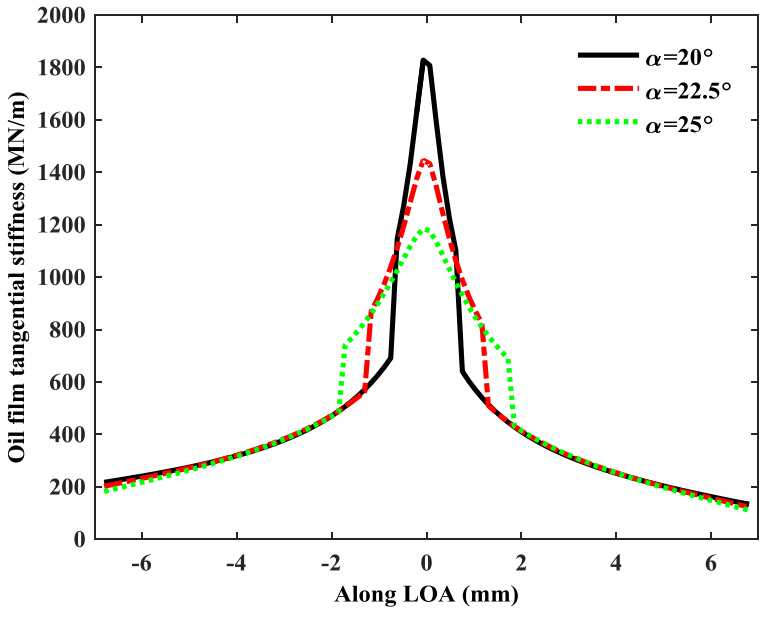

(b)

Fig. 11. Variation of the oil film normal and tangential stiffness with pressure angel

\subsection{Effect of modification}

It is shown in Fig. 12 that the oil film normal and tangential stiffness are related to the gear modification. In agreement with the load distribution in Fig.4, the oil film stiffness of tip relief or profile crown modification are larger than that of unmodified gear in double-teeth contact region, whereas the stiffness in single-tooth contact region has no change because the profile is not modified. Obviously, the jump of oil film stiffness at single-to-double and double-to-single tooth contact, which boosts the mesh impact and vibration, is diminished by the tip relief or profile crown modification. As a result, the modification is valid for inhibiting the jump of oil film stiffness and improving the stationarity of gear transmission.

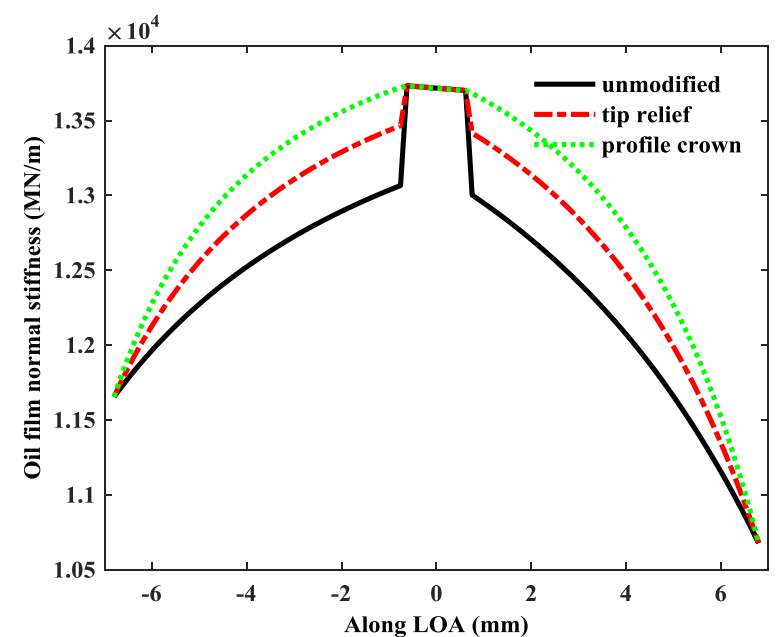

(a)

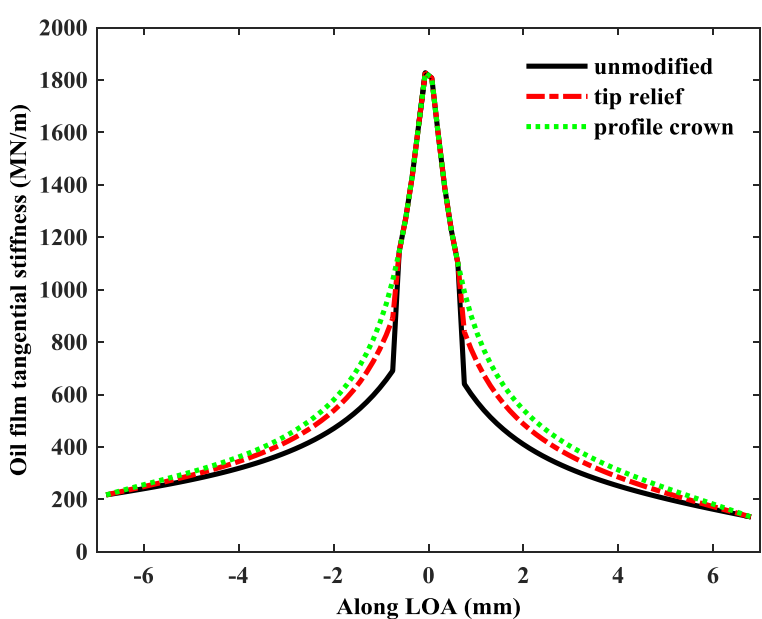

(b) 
Fig. 12. Variation of the oil film normal and tangential stiffness with modification

\section{Conclusions}

In this study, the new models of oil film normal and tangential stiffness were proposed based on a non-Newtonian EHL line contact for unmodified and modified spur gears, and the stiffness were calculated according to the relevant model and non-Newtonian EHL equations. According to the models, effects of operating parameters (contact force and rotation speed) and geometry parameters (module, tooth number, pressure angel and modification) on oil film stiffness along LOA from the root to the tip were investigated. Conclusions are summarized as follows:

(1) The oil film normal stiffness model is established by viscous-elastic fluid between the meshing teeth, equivalent to a massless spring element. And the stiffness is defined by the ratio of the summation to oil pressure increment and mean compression increment of the film thickness in transient contact domain. Meanwhile, the tangential stiffness model is built according to the hypothesis of equal shear stress on laminar element surfaces, where the minimal shear stress gradient across the oil film direction is neglected. Then the tangential stiffness is defined by the ratio of the summation to shear force increment on the film and mean increment of its tangential displacement in transient contact domain.

(2) The amplitude and fluctuation of oil film normal and tangential stiffness vary with the operating and geometry parameters. For a spur gear drive, the amplitude of film stiffness becomes smaller as increase of the geometry parameter values, and the stiffness fluctuation at single-to-double and double-to-single tooth contact is diminished by modification. Actually, the less amplitude and fluctuation of the oil film stiffness are valid in mesh impact reduction and stationarity enhancement. In addition, the oil film stiffness becomes larger as increase of the load increment, and the tangential stiffness is up as rise of rotation speed, whereas the normal stiffness falls down. Moreover, a sharp change of the tangential stiffness in single-tooth contact domain is caused by a little variation of operating or geometry parameter, which is mainly related to the effective viscosity mutation and non-Newtonian behavior reduction of the oil film near the pitch point. It is indicated that less loss of fluidity at single-tooth contact would be attained by rationally matching the operating and geometry parameters.

\section{Acknowledgments}

The authors gratefully acknowledge the support by the National Science Foundation of China (NSFC) through Grants Nos. 51675168 and 51275160, and by Open Research Fund of Key Laboratory of High Performance Complex Manufacturing, Central South University Kfkt2014-03.

\section{References}

[1] Xie G, Guo D, Luo J. Lubrication under charged conditions. Tribol Int, 2015, 84:22-35.

[2] Wu S, Zuo M J, Parey A. Simulation of spur gear dynamics and estimation of fault growth. J Sound Vib, 2008, 317(3-5):608-624.

[3] Chen S, Tang J, Hu Z. Comparisons of gear dynamic responses with rectangular mesh stiffness and its approximate form. J Mech Sci Technol, 2015, 29(9):3563-3569.

[4] Dowson D, Higginson G R. A numerical solution to the elasto-hydrodynamic problem. J Mech Eng Sci, 1959, 1(1):6-15.

[5] Larsson R. Transient non-Newtonian elastohydrodynamic lubrication analysis of an involute spur gear. Wear, 1997, 207(1):67-73.

[6] Raisin J, Fillot N, Dureisseix D, et al. Characteristic times in transient thermal elastohydrodynamic line contacts. Tribol Int, 2015, 82(82):472-483.

[7] Khonsari M M, Hua D Y. Thermal Elastohydrodynamic Analysis Using a Generalized Non-Newtonian Formulation With Application to Bair-Winer Constitutive Equation. J Tribol, 1994, 116(1):37-46.

[8] Kumar P, Khonsari M M. On the role of lubricant rheology and piezo-viscous properties in line and point contact EHL. Tribol Int, 2009, 42(11-12):1522-1530.

[9] Kumar P, Khonsari M M. Effect of Starvation on Traction and Film Thickness in Thermo-EHL Line Contacts with Shear-Thinning Lubricants. Tribol Let, 2008, 32(3):171-177.

[10] Chapkov A D, Venner C H, Lubrecht A A. Roughness Amplitude Reduction under Non-Newtonian EHD Lubrication Conditions. Tribology \& Interface Engineering, 2006, 128(4):637-648.

[11] Liu H, Zhu C, Sun Z, et al. Starved lubrication of a spur gear pair. Tribol Int, 2015, 94:52-60.

[12] Li S, Kahraman A. A spur gear mesh interface damping model based on elastohydrodynamic contact behavior. International Journal 
of Powertrains, 2011, 1(1):4-21.

[13] Ankouni M, Lubrecht A A, Velex P. Modelling of damping in lubricated line contacts - Applications to spur gear dynamic simulations. ARCHIVE Proceedings of the Institution of Mechanical Engineers Part C Journal of Mechanical Engineering Science 1989-1996 (vols 203-210), 2016, 230(7).

[14] Venner C H. Central film thickness in EHL point contacts under pure impact revisited. Tribol Int, 2015, 100:1-6.

[15] Habchi W. Influence of thermo-mechanical properties of coatings on friction in elastohydrodynamic lubricated contacts. Tribol Int, 2015, 90:113-122.

[16] Thakre G D, Sharma S C, Harsha S P, et al. A theoretical study of Ionic liquid lubricated $\mu$-EHL line contacts considering surface texture. Tribol Int, 2015, 94:39-51.

[17] Masjedi M, Khonsari M M. On the effect of surface roughness in point-contact EHL: Formulas for film thickness and asperity load. Tribol Int, 2015, 82:228-244.

[18] Li S, Kahraman A. A tribo-dynamic model of a spur gear pair. J Sound Vib, 2013, 332(20):4963-4978.

[19] Wiegert B, Hetzler H, Seemann W. A simplified elastohydrodynamic contact model capturing the nonlinear vibration behaviour. Tribol Int, 2013, 59(2):79-89

[20] Qin W, Chao J, Duan L. Study on stiffness of elastohydrodynamic line contact. Mech Mach Theory, 2015, 86(86):36-47.

[21] Iii D J D, Sawyer W G. Lateral Contact Stiffness and the Elastic Foundation. Tribol Let, 2011, 41(1):17-21.

[22] Shi J, Cao X, Zhu H. Tangential Contact Stiffness of Rough Cylindrical Faying Surfaces Based on the Fractal Theory. J Tribol, 2014, 136(4):919-927.

[23] Zhang Y, Liu H, Zhu C, et al. Oil film stiffness and damping in an elastohydrodynamic lubrication line contact-vibration. J Mech Sci Technol, 2016, 30(7):3031-3039.

[24] Masjedi M, Khonsari M M. A study on the effect of starvation in mixed elastohydrodynamic lubrication. Tribol Int, 2015, 85:26-36.

[25] Yang P, Wen S. A Generalized Reynolds Equation for Non-Newtonian Thermal Elastohydrodynamic Lubrication. J Tribol, 1990, 112(4):631-636.

[26] Johnson K L, Tevaarwerk J L. Shear Behaviour of Elastohydrodynamic Oil Films. P Roy Soc A-Math Phy, 1977, 356(1685):215-236.

[27] Dowson D, Venner C H, Lubrecht A A. Multi-Level Methods in Lubrication. Proceedings of the Institution of Mechanical Engineers Part J Journal of Engineering Tribology, 2000(5):495.

[28] Li S, Kahraman A. A Transient Mixed Elastohydrodynamic Lubrication Model for Spur Gear Pairs. J Tribol, 2010, $132(1): 011501$.

[29] Habchi W. A numerical model for the solution of thermal elastohydrodynamic lubrication in coated circular contacts. Tribol Int, 2014, 73(5):57-68. 